\title{
Subregional Patterns of Preferential Striatal Dopamine Transporter Loss Differ in Parkinson Disease, Progressive Supranuclear Palsy, and Multiple-System Atrophy
}

\author{
Minyoung Oh ${ }^{1}$, Jae Seung Kim ${ }^{1}$, Ji Young Kim ${ }^{1}$, Kwang-Ho Shin ${ }^{1}$, Seol Hoon Park ${ }^{1}$, Hye Ok Kim ${ }^{1}$, Dae Hyuk Moon ${ }^{1}$, \\ Seung Jun $\mathrm{Oh}^{1}$, Sun Ju Chung ${ }^{2}$, and Chong Sik Lee ${ }^{2}$ \\ ${ }^{1}$ Department of Nuclear Medicine, Asan Medical Center, College of Medicine, University of Ulsan, Seoul, Korea; and ${ }^{2}$ Department of \\ Neurology, Asan Medical Center, College of Medicine, University of Ulsan, Seoul, Korea
}

Parkinson disease (PD), progressive supranuclear palsy (PSP), and multiple-system atrophy (MSA) are known to affect dopaminergic neurons of the brain stem and striatum with different preferential involvement. Here we investigated differences in striatal subregional dopamine transporter loss in PD, PSP, and MSA and assessed the diagnostic value of ${ }^{18} \mathrm{~F}$-fluorinated- $N-3-$ fluoropropyl-2- $\beta$-carboxymethoxy-3- $\beta$-(4-iodophenyl)nortropane ( $\left.{ }^{18} \mathrm{~F}-\mathrm{FP}-\mathrm{CIT}\right)$ PET in differentiating PSP and MSA from PD. Methods: Forty-nine patients with PD, 19 patients with PSP, 24 patients with MSA, and 21 healthy people (healthy controls) were examined with ${ }^{18} \mathrm{~F}-\mathrm{FP}-\mathrm{CIT}$ PET. The PET images were spatially normalized and analyzed with 12 striatal subregional volume-of-interest (VOI) templates (bilateral ventral striatum [VS], anterior caudate [AC], posterior caudate, anterior putamen, posterior putamen [PP], and ventral putamen [VP]) and 1 occipital VOI template. The nondisplaceable binding potential $\left(\mathrm{BP}_{\mathrm{ND}}\right)$ and intersubregional ratio (ISR; defined as the ratio of the $\mathrm{BP}_{\mathrm{ND}}$ of one striatal subregion to that of another striatal subregion) of subregional VOIs were calculated. Results: The $\mathrm{BP}_{\mathrm{ND}}$ of all VOls in the PD, MSA, and PSP groups were significantly lower than those in the healthy controls $(P<0.05)$. The $\mathrm{BP}_{\mathrm{ND}}$ of $A C$ and the AC/VS ISR in the PSP group were significantly lower than those in the PD group. The $\mathrm{BP}_{\mathrm{ND}}$ of VP was significantly lower, but the PP/VP ISR was significantly higher in the MSA group than in the PD group. At the cutoff value for the AC/VS ISR $(<0.7)$, the sensitivity and specificity for differentiating PSP from PD were $94 \%$ and $92 \%$, respectively. At the cutoff value for the PP/VP ISR $(>0.65)$, the sensitivity and specificity for differentiating MSA from PD were $90 \%$ and $45 \%$, respectively. The diagnostic accuracy of visual analysis was similar to that of quantitative analysis for differentiating PSP from PD but was significantly higher for differentiating MSA from PD. Conclusion: Compared with PD, PSP and MSA showed more prominent and earlier dopamine transporter loss in the AC and VP, respectively. These findings could be useful for suggesting PSP or MSA in parkinsonian cases without characteristic atypical features.

\footnotetext{
Received Jun. 30, 2011; revision accepted Oct. 17, 2011.

For correspondence or reprints contact: Jae Seung Kim, Department of Nuclear Medicine, Asan Medical Center, College of Medicine, University of Ulsan, 88, Olympic-ro 43-gil, Songpa-gu, Seoul 138-736, Korea.

E-mail: jaeskim@amc.seoul.kr

Published online Feb. 9, 2012

COPYRIGHT @ 2012 by the Society of Nuclear Medicine, Inc.
}

Key Words: atypical parkinsonism; ${ }^{18} \mathrm{~F}-\mathrm{FP}-\mathrm{CIT}$; PET; striatum; subregion

J Nucl Med 2012; 53:399-406

DOI: 10.2967/jnumed.111.095224

$\mathbf{P}$ arkinsonian disorders have clinically similar features characterized by akinesia with resting tremor, rigidity, or postural instability (1) but exhibit pathologically distinct features (2). Idiopathic Parkinson disease (PD), the most common, is slowly progressive and responsive to L-3,4-dihydroxyphenylalanine. Atypical parkinsonian disorders (APD), such as progressive supranuclear palsy (PSP) and multiple-system atrophy (MSA), which also account for a significant proportion of parkinsonian cases (3), exhibit a rapidly evolving and fatal neurodegenerative course $(4,5)$. Nevertheless, the majority of patients with APD have clinical features that overlap those of PD. A major concern in differentiating parkinsonian disorders is early diagnosis, particularly at a stage of disease evolution when intervention is likely to be most effective. Consequently, making an accurate diagnosis is critical, not only for choosing treatment regimens (6) and assessing prognosis (7) but also for understanding pathogenesis and developing new therapeutic strategies (8). Although the definition of PD is in an active state of evolution, it mainly requires the histologic demonstration of intraneuronal Lewy body inclusions in the nervous system (9) - a test that is clearly impractical during a patient's life. The application of less sensitive clinical criteria (10) based on retrospective clinicopathologic studies only intensifies the confusion. Consequently, the diagnosis of parkinsonian disorders continues to be challenging, with misdiagnosis rates of $20 \%-30 \%$ in early stages (11).

Numerous efforts have been made to improve the diagnostic accuracy of tests used to discriminate APD from PD. Functional imaging techniques such as both pre- and postsynaptic dopaminergic SPECT or PET and ${ }^{18}$ F-FDG PET have been studied for their potential ability to differentiate APD from PD $(12,13)$. 
To date, dopamine receptor imaging and ${ }^{18} \mathrm{~F}-\mathrm{FDG}$ PET have shown promise in distinguishing among parkinsonian disorders. Presynaptic dopamine transporter (DAT) imaging, in contrast, has not proved as useful as dopamine receptor or ${ }^{18} \mathrm{~F}-\mathrm{FDG}$ imaging in differentiating APD from $\mathrm{PD}$, although it is useful in ruling out essential tremor, drug-induced parkinsonism, vascular parkinsonism, and Alzheimer disease because it reveals significant striatal DAT loss in both PSP and MSA as well as PD $(14,15)$.

${ }^{18} \mathrm{~F}$-fluorinated- $N$-3-fluoropropyl-2- $\beta$-carboxymethoxy-3$\beta$-(4-iodophenyl)nortropane ( ${ }^{18} \mathrm{~F}$-FP-CIT) PET/CT, which has recently become available for DAT imaging in clinical practice, has better spatial resolution and more sophisticated attenuation correction than SPECT (16). Thus, it is possible to use ${ }^{18} \mathrm{~F}$-FP-CIT PET to evaluate subregional changes in the striatal DAT (17) and to differentiate parkinsonian disorders on the basis of the more asymmetric and pronounced loss of the posterior putamen (PP) in PD than in APD $(14,15)$.

The purposes of this study were to investigate differences in subregional DAT loss in PD, PSP, and MSA and to establish the diagnostic value of ${ }^{18} \mathrm{~F}$-FP-CIT PET in differentiating PSP and MSA from PD.

\section{MATERIALS AND METHODS}

\section{Subjects}

Nineteen patients with clinically probable PSP, 24 patients with clinically probable MSA, and 49 patients with PD at various Hoehn-Yahr stages were enrolled in this study. The diagnosis of PD was based on UK Parkinson's Disease Society Brain Bank Clinical Diagnostic Criteria (18). Patients with clinically probable PSP (the classic Richardson syndrome) and MSA (parkinsonian type) were enrolled on the basis of current diagnostic criteria $(4,19)$. All patients were assessed by a neurologist specializing in movement disorders. Their medical records were available. The severity of motor symptoms was evaluated with the motor portion of the Unified Parkinson Disease Rating Scale, Part III (UPDRS III), immediately before PET and after antiparkinsonian drugs had been discontinued for at least $12 \mathrm{~h}$ (considered "off medication").

Twenty-one healthy people (healthy controls) ( $>40$ y old) were selected from among a normal ${ }^{18} \mathrm{~F}$-FP-CIT PET data pool maintained at our institution. Healthy controls had no neurologic or psychologic diseases. This study was approved by the institutional review board, and written informed consent was obtained from each subject.

\section{Radiopharmaceutical Synthesis}

${ }^{18} \mathrm{~F}$-FP-CIT was synthesized with a protic solvent ( $t$-butanol or $t$-amyl alcohol) as a reaction solvent and $N$-[3'-(tosyloxy)propyl]$2 \beta$-carbomethoxy-3 $\beta$-(4'-iodophenyl)nortropane as a precursor (20). The radiochemical yield (mean $\pm \mathrm{SD}$ ) was $42.5 \% \pm 10.9 \%$ (decay corrected), the radiochemical purity was greater than $98 \%$ after purification by high-performance liquid chromatography, and the specific activity was $64.4 \pm 4.5 \mathrm{GBq} / \mu \mathrm{mol}$ at the end of synthesis.

\section{PET/CT}

${ }^{18}$ F-FP-CIT PET was performed with a Biograph 40 TruePoint PET/CT camera (Siemens/CTI), which provides an in-plane spatial resolution of $2.0 \mathrm{~mm}$ full width at half maximum at the center of the field of view. All subjects had fasted for at least $6 \mathrm{~h}$, and antiparkinsonian drugs had been stopped $12 \mathrm{~h}$ before the scans were obtained. Image acquisition was started $3 \mathrm{~h}$ after intravenous injection of ${ }^{18}$ F-FP-CIT (185 MBq). Emission PET data were acquired for $10 \mathrm{~min}$ in the 3-dimensional mode after brain CT, which was performed in the spiral mode at $120 \mathrm{kVp}$ and 380 $\mathrm{mAs}$ (reference standard) with the CARE Dose $4 \mathrm{D}$ program. ${ }^{18} \mathrm{~F}$ FP-CIT PET images were reconstructed from CT data for attenuation correction by use of the TrueX algorithm and an all-pass filter with a $336 \times 336$ matrix.

\section{Quantitative Analyses}

Image processing was performed with SPM2 (Wellcome Department of Imaging Neuroscience, Institute of Neurology, University College London) within MATLAB 6.5.1 for Windows (The MathWorks, Inc.) and MRIcro version 1.37 (Chris Rorden, Columbia, SC, USA. www.mricro.com).

Quantitative analyses were based on volumes of interest (VOIs), which were defined on the basis of a template in standard space. All reconstructed PET images were spatially normalized to Talairach space by use of a standard ${ }^{18}$ F-FP-CIT PET template, which was made in-house from ${ }^{18} \mathrm{~F}$-FP-CIT PET and T1-weighted MR images of 13 healthy controls (4 men and 9 women; $55.2 \pm$ 9.2 y old; age range, $41-70$ y) as described previously to remove intersubject anatomic variability (21). Images of patients were reoriented so that the striatum contralateral to the symptomatic side was on the left side of the brain. If no laterality was present, then the anatomic left became the more affected side.

Twelve VOIs of bilateral striatal subregions and one occipital VOI were drawn manually on a coregistered, spatially normalized, single T1-weighted MRI and ${ }^{18} \mathrm{~F}-\mathrm{FP}-\mathrm{CIT}$ PET template image by an experienced nuclear medicine physician specializing in nuclear neurology. The VOI for the ventral striatum (VS) was defined on the basis of previously defined criteria (22); the caudate nucleus and putamen were further divided into 5 subregions: anterior caudate (AC), posterior caudate (PC), anterior putamen (AP), PP, and ventral putamen (VP). The boundary point between the AC and the $\mathrm{PC}$ and between the AP and the PP was the anterior commissure coronal plane. The boundary point between the PP and the VP was the anterior-posterior commissure transaxial plane. The outer boundaries of the striatal subregions were visually determined from the characteristic increased activity of the striatum, which readily distinguished these subregions from extrastriatal structures (Fig. 1).

The VOI template in the standard stereotactic space was automatically applied directly to the spatially normalized individual PET images to analyze striatal ${ }^{18} \mathrm{~F}$-FP-CIT binding.

The activity concentration in each VOI was calculated. The nondisplaceable binding potential $\left(\mathrm{BP}_{\mathrm{ND}}\right)$ was defined as follows: (mean standardized uptake value [SUV] of striatal subregional VOI - mean SUV of occipital VOI)/mean SUV of occipital VOI. The age-corrected normalized $\mathrm{BP}_{\mathrm{ND}}(\% \mathrm{BP})$ was defined as follows: $100 \times\left(\mathrm{BP}_{\mathrm{ND}}\right.$ for patient $/ \mathrm{BP}_{\mathrm{ND}}$ for age-matched healthy control). The intersubregional ratio (ISR) was defined as the ratio of the $\mathrm{BP}_{\mathrm{ND}}$ of one striatal subregion to that of another striatal subregion. The ISRs for the anteroposterior gradient (AP/PP, AC/PC, and PP/AC), the ventrodorsal gradient (AC/VS, AP/VS, AC/AP, PP/VP, and PC/VP), and the asymmetry of the $\mathrm{BP}_{\mathrm{ND}}$ for each of the 6 striatal subregions were calculated.

\section{Visual Analysis}

For visual interpretation, ${ }^{18} \mathrm{~F}-\mathrm{FP}$-CIT PET/CT images were displayed as a series of 148 transaxial slices scaled to a common 


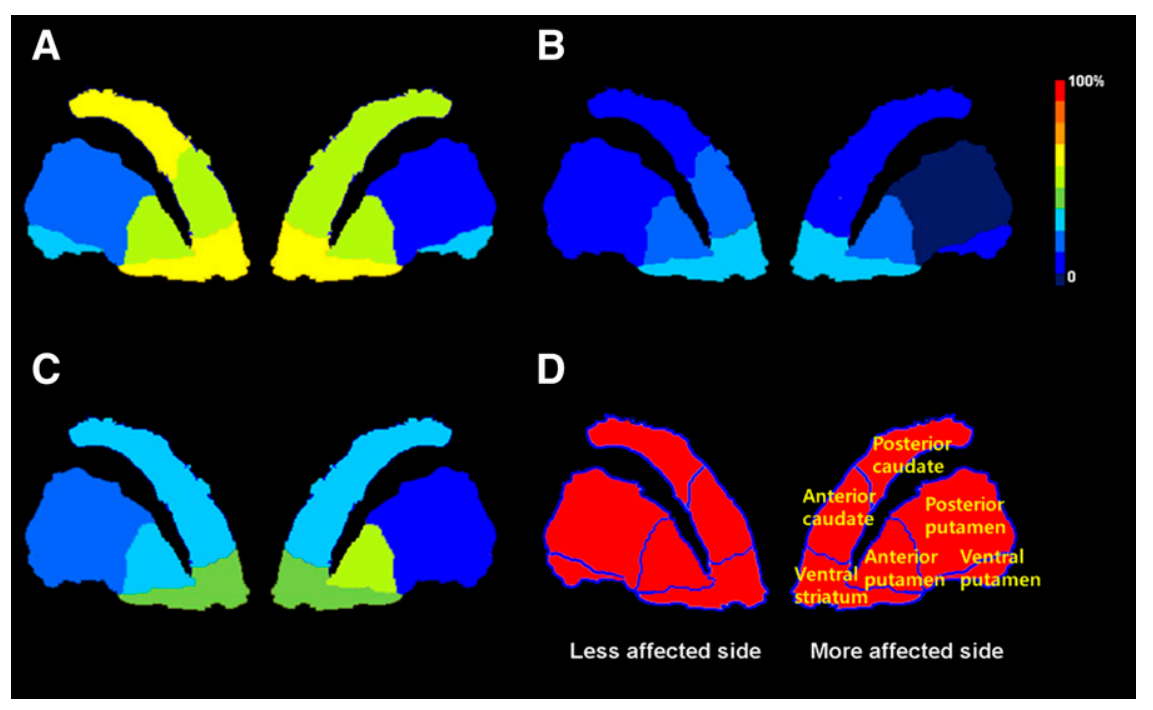

FIGURE 1. Diagram of age-corrected, normalized \%BP of striatal subregions on ${ }^{18} \mathrm{~F}-$ FP-CIT PET for PD (A), PSP (B), MSA (C), and healthy controls (D). maximum in a standard color scale on a dedicated workstation (Singo; Siemens). All PET images were compared visually. First, they were independently classified as PD, PSP, or MSA; next, they were assigned as PSP (or MSA) versus PD by 3 nuclear medicine physicians who were completely unaware of the clinical information. A training session had been organized for learning typical images (Fig. 2) of each disease entity on the basis of the results of the VOI analysis.

\section{Statistical Analyses}

Continuous variables among groups were compared with 1-way ANOVAs. The Bonferroni correction was applied to a post hoc analysis of between-group comparisons. For multiple comparisons of $\mathrm{BP}_{\mathrm{ND}}$ and $\% \mathrm{BP}$, a $P$ value of less than $0.008(0.05 / 6)$ was chosen. For ISRs, a $P$ value of less than $0.004(0.05 / 12)$ was chosen. For comparisons of $\% \mathrm{BP}$ among groups, $\mathrm{BP}_{\mathrm{ND}}$ values were age corrected by fitting each patient's data to a regression line calculated from a sample of 21 healthy controls and were expressed as percentages of control values for each striatal subregion as previously reported (23). A receiver-operatingcharacteristic curve analysis was used to select the most significant ISR for discriminating APD from PD. Pearson correlation analyses were used to identify the correlations between ISRs and clinical parameters. For visual analysis, agreement was measured between

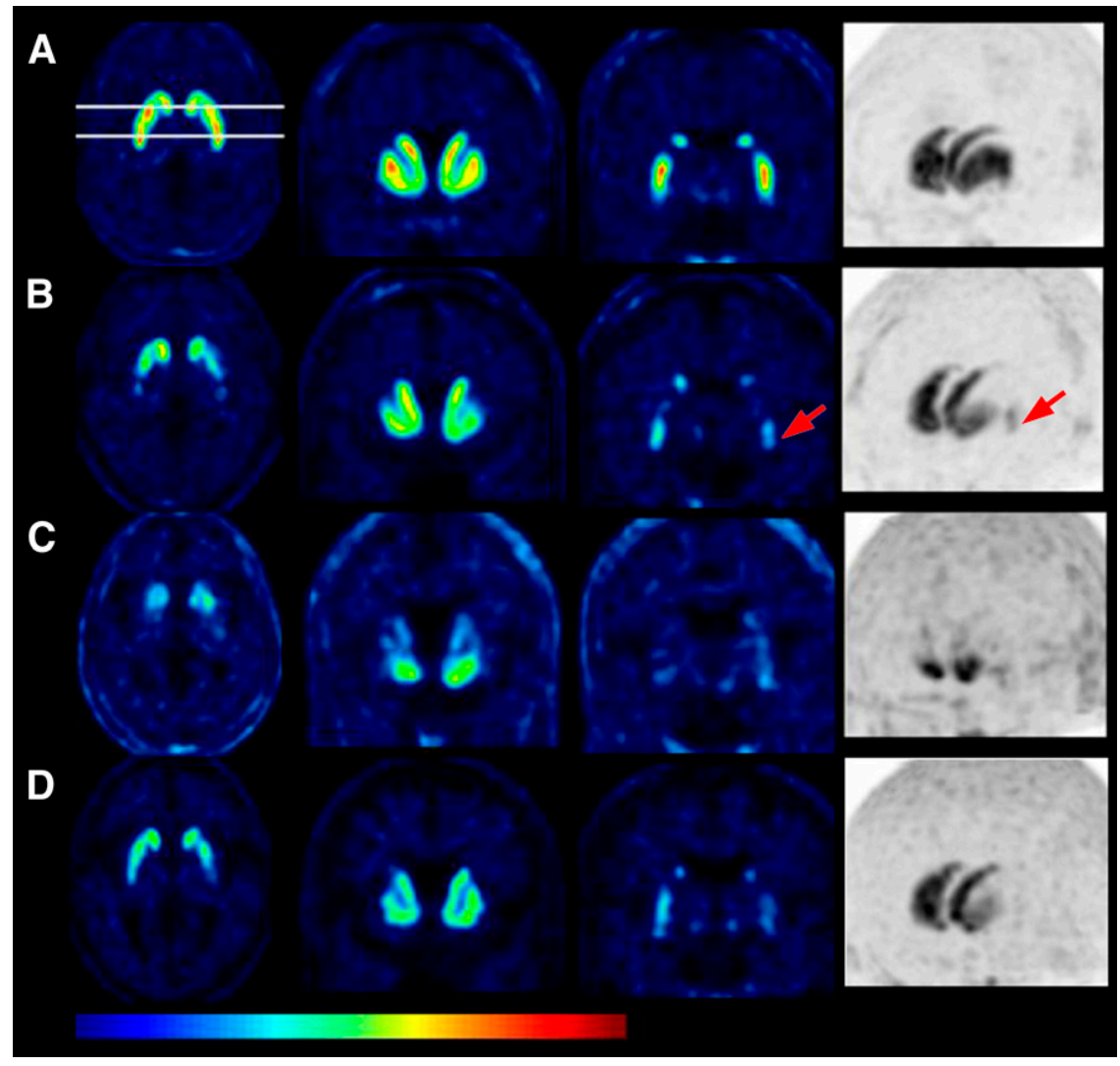

FIGURE 2. Representative ratio (regional VOI to occipital VOI) parametric axial images at level of anterior-posterior commissure, coronal image at level $7 \mathrm{~mm}$ anterior to anterior commissure, coronal image at level $17 \mathrm{~mm}$ posterior to anterior commissure, and maximum-intensity-projection image from ${ }^{18} \mathrm{~F}-\mathrm{FP}$-CIT PET of healthy controls (A) and PD (B), PSP (C), and MSA (D) groups. (B) PET images of patients with PD showed typical preferential DAT loss in dorsal PP. Note relative sparing of DAT loss in VP (arrow). (C) PET images of patients with PSP showed preferential DAT loss in caudate nuclei as well as in putamen. (D) PET images of patients with MSA showed preferential DAT loss in VP as well as in dorsal PP. 
readers, and the Fleiss $\kappa$ coefficient was used to evaluate overall agreement. The McNemar test was used to compare significant differences between quantitative analysis and visual analysis. A $P$ value of less than 0.05 was considered statistically significant.

Data for the study variables were expressed as mean $\pm \mathrm{SD}$. We used SPSS version 17.0 (SPSS Inc.) and SAS 9.1 (SAS Institute Inc.) software.

\section{RESULTS}

The clinical characteristics of the patients with PD, PSP, and MSA and the healthy controls are summarized in Table 1. There were no significant differences in sex, duration of symptoms, or UPDRS III scores among the disease groups. Patients in the PSP and MSA groups had more advanced Hoehn-Yahr stages than patients in the PD group $(P<$ 0.001 for both PSP and MSA).

\section{Vol Analysis}

Data from 5 cases (1 PD, 2 PSP, and 2 MSA) for which ${ }^{18}$ F-FP-CIT PET images showed visually evident misregistration with predefined VOI templates after spatial normalization were excluded from the VOI analysis.

$\mathrm{BP}_{\mathrm{ND}}$ and age-corrected \% $\mathrm{BP}$ values for all bilateral striatal subregions in the PD, PSP, and MSA groups were significantly lower than those in the healthy controls $(P<$ 0.001) (Table 2 and Fig. 2). A post hoc analysis revealed that $\mathrm{BP}_{\mathrm{ND}}$ and age-corrected $\% \mathrm{BP}$ values for the whole striatum and several subregions in the PSP group were significantly lower than those in the PD group $(P<0.001)$.

The ISRs for the anteroposterior gradient, ventrodorsal gradient, and asymmetry in the PD, PSP, and MSA groups and healthy controls are shown in Tables 3 and 4. The PP/ AC ISR (anteroposterior gradient) in the PD group was significantly lower than those in the healthy controls and the PSP group. The asymmetry of striatal $\mathrm{BP}_{\mathrm{ND}}$ values was significantly different only for PP in the PD group and the healthy controls $(P<0.001)$.

Among the ISRs suggesting a ventrodorsal gradient, the AC/VS ISR in the PSP group was significantly lower than that in the PD group $(0.5 \pm 0.2$ vs. $1.0 \pm 0.2$ on the more affected side $[P<0.001] ; 0.5 \pm 0.3$ vs. $1.0 \pm 0.2$ on the less affected side $[P<0.001]$ ) (Fig. 3A). The AC/VS ISR correlated positively with the UPDRS III score and symptom duration in the PD group $(P<0.05)$ but not in the PSP group (Supplemental Fig. 1) (supplemental materials are available online only at http://jnm.snmjournals.org). A receiver-operating-characteristic curve analysis showed that the AC/VS ISR on the more affected side had the largest area under the curve ( 0.958 and 0.928 on the more and less affected sides, respectively) and was the most significant parameter for discriminating PSP from PD. At the cutoff value for the AC/VS ISR $(<0.7)$, the sensitivity, specificity, positive predictive value, and negative predictive value for differentiating PSP from PD were 94\%, 92\%, 80\%, and $98 \%$, respectively.

Among the ISRs suggesting a ventrodorsal gradient, the PP/VP ISR in the MSA group was significantly higher than that in the PD group on the more affected side $(0.8 \pm 0.3$ vs. $0.6 \pm 0.3[P=0.002]$ ) (Fig. $3 \mathrm{~B}$ ). The PP/VP ISR correlated positively with the UPDRS III score and symptom duration in the PD group $(P<0.01)$ but not in the MSA group (Supplemental Fig. 2). A receiver-operatingcharacteristic curve analysis revealed that the PP/VP ISR on the less affected side had the largest area under the curve ( 0.709 and 0.736 on the more and less affected sides, respectively) and was the most significant parameter for discriminating MSA from PD. At the cutoff value for the PP/ VP ISR $(>0.65)$, the sensitivity, specificity, positive predictive value, and negative predictive value for differentiating MSA from PD were $90 \%, 45 \%, 44 \%$, and $92 \%$, respectively.

\section{Visual Analysis}

On axial, coronal, and maximum-intensity-projection images of ${ }^{18} \mathrm{~F}$-FP-CIT PET, patients with PD showed a preferential decrease in DAT binding in the PP, with relative sparing of the VP ("rabbit hip" sign on the maximum-intensity-projection image). Patients with PSP showed a preferential decrease in DAT binding in the caudate nuclei ("rabbit ear" sign) as well as in the putamen. Patients with MSA showed a preferential decrease in DAT binding in the VP ("rabbit foot" sign) as well as in the PP (Fig. 2).

The results of the visual analysis are shown in Table 5 . The interobserver agreement of 3 interpreters in the visual

TABLE 1

Clinical Characteristics of Subjects

\begin{tabular}{|c|c|c|c|c|}
\hline Characteristic & PD group $(n=49)$ & PSP group $(n=19)$ & MSA group $(n=24)$ & Healthy controls $(n=21)$ \\
\hline Age $(y)$ & $62 \pm 11$ & $68 \pm 8$ & $62 \pm 11$ & $61 \pm 12$ \\
\hline $\operatorname{Sex}(\mathrm{M} / \mathrm{F}), n$ & $21 / 28$ & $9 / 10$ & $8 / 16$ & $6 / 15$ \\
\hline Symptom duration (y) & $5.1 \pm 6.0$ & $3.9 \pm 2.1$ & $3.0 \pm 1.7$ & NA \\
\hline Hoehn-Yahr stage & $2.0 \pm 1.0$ & $3.4 \pm 1.3^{*}$ & $4.0 \pm 1.2^{\dagger}$ & NA \\
\hline UPDRS III score & $20.4 \pm 13.3$ & $25.5 \pm 12.7$ & $35.4 \pm 16.5$ & NA \\
\hline \multicolumn{5}{|c|}{$\begin{array}{l}{ }^{\star} P<0.05 \text { for PD vs. PSP. } \\
{ }^{\dagger} P<0.05 \text { for PD vs. MSA. } \\
\text { NA }=\text { not applicable. } \\
\text { Values are reported as mea }\end{array}$} \\
\hline
\end{tabular}


TABLE 2

$\mathrm{BP}_{\mathrm{ND}}$ of Striatal Subregions in Healthy Controls and PD, PSP, and MSA Groups

\begin{tabular}{|c|c|c|c|c|c|c|c|}
\hline \multirow[b]{2}{*}{ Region } & \multirow[b]{2}{*}{ Side $^{*}$} & \multicolumn{4}{|c|}{$\mathrm{BP}_{\mathrm{ND}}($ mean $\pm \mathrm{SD})$ in: } & \multicolumn{2}{|c|}{$P$ for: } \\
\hline & & Healthy controls & PD group & PSP group & MSA group & PD vs. PSP & PD vs. MSA \\
\hline \multirow[t]{2}{*}{ Whole striatum } & More affected & $6.4 \pm 0.9$ & $2.4 \pm 1.1$ & $1.1 \pm 0.4$ & $1.7 \pm 0.8$ & $<0.001$ & NS \\
\hline & Less affected & & $3.1 \pm 1.4$ & $1.3 \pm 0.8$ & $2.2 \pm 1.2$ & $<0.001$ & NS \\
\hline \multicolumn{8}{|l|}{ Subregion } \\
\hline \multirow[t]{2}{*}{$A C$} & More affected & $7.4 \pm 1.1$ & $3.7 \pm 1.8$ & $1.1 \pm 0.6$ & $2.3 \pm 1.3$ & $<0.001$ & 0.002 \\
\hline & Less affected & & $4.0 \pm 1.7$ & $1.3 \pm 0.9$ & $2.7 \pm 1.4$ & $<0.001$ & 0.003 \\
\hline \multirow[t]{2}{*}{$\mathrm{PC}$} & More affected & $5.2 \pm 0.9$ & $2.7 \pm 1.8$ & $0.5 \pm 0.4$ & $1.5 \pm 1.1$ & $<0.001$ & 0.003 \\
\hline & Less affected & & $3.1 \pm 1.9$ & $0.5 \pm 0.5$ & $1.8 \pm 1.2$ & $<0.001$ & 0.004 \\
\hline \multirow[t]{2}{*}{ VS } & More affected & $6.0 \pm 0.9$ & $3.6 \pm 1.3$ & $2.3 \pm 0.9$ & $2.6 \pm 1.2$ & 0.001 & 0.007 \\
\hline & Less affected & & $3.8 \pm 1.2$ & $2.3 \pm 1.2$ & $2.8 \pm 1.1$ & $<0.001$ & 0.003 \\
\hline \multirow[t]{2}{*}{ AP } & More affected & $8.1 \pm 1.3$ & $2.7 \pm 1.6$ & $1.5 \pm 0.7$ & $2.2 \pm 1.2$ & NS & NS \\
\hline & Less affected & & $3.4 \pm 1.8$ & $1.7 \pm 0.9$ & $2.5 \pm 1.4$ & 0.001 & NS \\
\hline \multirow[t]{2}{*}{ PP } & More affected & $7.7 \pm 1.2$ & $1.4 \pm 1.2$ & $0.6 \pm 0.5$ & $1.1 \pm 1.0$ & NS & NS \\
\hline & Less affected & & $1.8 \pm 1.4$ & $0.8 \pm 0.6$ & $1.6 \pm 1.4$ & NS & NS \\
\hline \multirow[t]{2}{*}{ VP } & More affected & $7.1 \pm 1.1$ & $2.0 \pm 1.0$ & $0.9 \pm 0.4$ & $1.3 \pm 0.9$ & $<0.001$ & 0.007 \\
\hline & Less affected & & $2.4 \pm 1.3$ & $1.0 \pm 0.6$ & $1.7 \pm 1.2$ & $<0.001$ & NS \\
\hline
\end{tabular}

${ }^{*}$ Does not apply to values for healthy controls.

$\mathrm{NS}=$ not significant.

analysis was good (Fleiss $\kappa$ coefficient, $0.70[P<0.001])$. The rates of concordance of clinical and visual diagnoses were $96 \%$ for PD, $78 \%$ for PSP, and $67 \%$ for MSA.

The sensitivity, specificity, positive predictive value, and negative predictive value for discriminating PSP from PD were $84 \%, 98 \%, 94 \%$, and 94\%, respectively; for discriminating MSA from PD, the respective values were $79 \%$, $96 \%, 90 \%$, and $90 \%$. The diagnostic accuracy of visual analysis was similar to that of quantitative analysis for differentiating PSP from PD $(P=0.250)$ but was significantly higher for differentiating MSA from PD $(P<0.001)$.

\section{DISCUSSION}

In the present study, all groups of patients showed significantly reduced striatal subregional $\mathrm{BP}_{\mathrm{ND}}$ values, in- dicative of presynaptic degeneration, but exhibited different preferential subregional losses of striatal DAT on ${ }^{18} \mathrm{~F}-\mathrm{FP}-$ CIT PET. Compared with patients with PD, patients with PSP and MSA showed more prominent and earlier DAT losses in the AC and VP, respectively.

The striatum is divided into 3 functional parts: the VS, which is strongly implicated in emotion, motivation, and reward-guided behaviors; the associative striatum, which is largely involved in cognition; and the sensorimotor striatum, which is involved in motor function (24). The striatum was further divided into more substructures-VS, VP, AP, $\mathrm{PP}, \mathrm{AC}$, and $\mathrm{AP}$-in the present study than in previous studies $(17,25)$ because of the higher resolution offered by a new-generation PET scanner (26). This scenario enabled us to understand the complex functional anatomy of

TABLE 3

ISRs for Gradients of $\mathrm{BP}_{\mathrm{ND}}$ in PD, PSP, and MSA Groups

\begin{tabular}{|c|c|c|c|c|c|c|c|}
\hline \multirow[b]{3}{*}{ Gradient } & \multicolumn{7}{|c|}{ ISR for $\mathrm{BP}_{\mathrm{ND}}($ mean $\pm \mathrm{SD})$ in: } \\
\hline & \multirow[b]{2}{*}{$\begin{array}{l}\text { Healthy } \\
\text { controls }\end{array}$} & \multicolumn{2}{|c|}{ PD group } & \multicolumn{2}{|c|}{ PSP group } & \multicolumn{2}{|c|}{ MSA group } \\
\hline & & $\begin{array}{l}\text { More affected } \\
\text { side }\end{array}$ & $\begin{array}{l}\text { Less affected } \\
\text { side }\end{array}$ & $\begin{array}{l}\text { More affected } \\
\text { side }\end{array}$ & $\begin{array}{l}\text { Less affected } \\
\text { side }\end{array}$ & $\begin{array}{l}\text { More affected } \\
\text { side }\end{array}$ & $\begin{array}{l}\text { Less affected } \\
\text { side }\end{array}$ \\
\hline \multicolumn{8}{|c|}{ Anteroposterior } \\
\hline PP/AP & $0.9 \pm 0.0$ & $0.5 \pm 0.2$ & $0.5 \pm 0.2$ & $0.4 \pm 0.3$ & $0.4 \pm 0.2$ & $0.5 \pm 0.2$ & $0.6 \pm 0.2$ \\
\hline $\mathrm{PC} / \mathrm{AC}$ & $0.7 \pm 0.0$ & $0.7 \pm 0.2$ & $0.7 \pm 0.2$ & $0.3 \pm 0.2$ & $0.4 \pm 0.3$ & $0.7 \pm 0.3$ & $0.7 \pm 0.3$ \\
\hline PP/AC & $1.1 \pm 0.2$ & $0.3 \pm 0.2$ & $0.7 \pm 0.2$ & $0.7 \pm 0.8$ & $0.7 \pm 0.2$ & $0.5 \pm 0.4$ & $0.7 \pm 0.2$ \\
\hline \multicolumn{8}{|l|}{ Ventrodorsal } \\
\hline AC/VS & $1.2 \pm 0.1$ & $1.0 \pm 0.2$ & $1.0 \pm 0.2$ & $0.5 \pm 0.2$ & $0.5 \pm 0.3$ & $0.8 \pm 0.2$ & $0.9 \pm 0.3$ \\
\hline AP/VS & $1.4 \pm 0.0$ & $0.7 \pm 0.3$ & $0.8 \pm 0.3$ & $0.7 \pm 0.2$ & $0.7 \pm 0.3$ & $0.8 \pm 0.2$ & $0.9 \pm 0.2$ \\
\hline $\mathrm{AC} / \mathrm{AP}$ & $0.9 \pm 0.1$ & $1.7 \pm 1.1$ & $1.7 \pm 2.4$ & $0.7 \pm 0.4$ & $0.7 \pm 0.4$ & $1.1 \pm 0.4$ & $1.2 \pm 0.7$ \\
\hline PP/NP & $1.1 \pm 0.0$ & $0.6 \pm 0.3$ & $0.7 \pm 0.2$ & $0.6 \pm 0.3$ & $0.7 \pm 0.4$ & $0.8 \pm 0.3$ & $0.9 \pm 0.2$ \\
\hline $\mathrm{PC} / \mathrm{PP}$ & $0.7 \pm 0.1$ & $1.2 \pm 0.5$ & $1.2 \pm 0.4$ & $0.5 \pm 0.4$ & $0.5 \pm 0.4$ & $1.2 \pm 0.6$ & $1.2 \pm 0.7$ \\
\hline
\end{tabular}


TABLE 4

ISRs for Asymmetry (Left to Right) of $\mathrm{BP}_{\mathrm{ND}}$ in PD, PSP, and MSA Groups

\begin{tabular}{|c|c|c|c|c|}
\hline \multirow[b]{2}{*}{$\begin{array}{l}\text { Striatal } \\
\text { subregion }\end{array}$} & \multicolumn{4}{|c|}{ ISR for $\mathrm{BP}_{\mathrm{ND}}($ mean $\pm \mathrm{SD})$ in: } \\
\hline & $\begin{array}{l}\text { Healthy } \\
\text { controls }\end{array}$ & PD group & $\begin{array}{l}\text { PSP } \\
\text { group }\end{array}$ & $\begin{array}{l}\text { MSA } \\
\text { group }\end{array}$ \\
\hline$A C$ & $1.0 \pm 0.0$ & $0.9 \pm 0.1$ & $0.9 \pm 0.5$ & $0.9 \pm 0.4$ \\
\hline PC & $0.9 \pm 0.1$ & $0.9 \pm 0.2$ & $1.5 \pm 2.8$ & $1.0 \pm 0.5$ \\
\hline VS & $1.0 \pm 0.0$ & $1.0 \pm 0.2$ & $1.1 \pm 0.4$ & $1.0 \pm 0.2$ \\
\hline $\mathrm{AP}$ & $1.0 \pm 0.0$ & $0.8 \pm 0.4$ & $1.2 \pm 0.7$ & $0.9 \pm 0.2$ \\
\hline PP & $1.1 \pm 0.0$ & $0.7 \pm 0.2$ & $0.9 \pm 0.6$ & $0.9 \pm 0.4$ \\
\hline VP & $1.0 \pm 0.1$ & $0.9 \pm 0.1$ & $1.3 \pm 1.5$ & $0.9 \pm 0.3$ \\
\hline
\end{tabular}

this structure, which is crucial to understanding the pathophysiology of parkinsonian disorders. For example, we were able to observe that DAT binding was always mostly spared in the VS in all groups of patients (Fig. 2). This sparing of the VS relative to the associative striatum or the sensorimotor striatum could have implications in the context of the anatomic framework described earlier.

Our results showed an anteroposterior gradient in PD, indicating greater involvement in the PP than in the AP, especially on the more affected side. These results are consistent with previous ${ }^{123} \mathrm{I}-\beta$-CIT studies $(17,27)$. However, the relative sparing of DAT loss in the VP in PD resulted in a ventrodorsal gradient of putaminal DAT loss. A previous study with ${ }^{18} \mathrm{~F}-3,4$-dihydroxyphenylalanine PET showed a gradient in dopamine metabolism from the ventral to the dorsal portion of the caudal putamen in patients with PD (27). This pattern may reflect the neuropathologic basis of $\mathrm{PD}$, in which the particularly early involvement of the ventrolateral part of the pars compacta of the substantia nigra, which projects to the posterior part of the putamen, is known to occur (28). Our results also supported the findings that the mean density of $\alpha$-synuclein immunoreactivity was higher in the lateral segment of the substantia nigra and inversely correlated with the density of DAT immunoreactivity in brains with synucleinopathy (29). The preservation of DAT binding in the VP in PD is reasonable, considering the role of the VS.

Patients with PSP showed the most extensive and symmetric pattern of striatal DAT loss, including that in the caudate nuclei, on ${ }^{18} \mathrm{~F}$-FP-CIT PET. These results are supported by a pathology study that found a more widespread loss of dopamine neurons in PSP than in PD (30). In terms of the ventrodorsal gradient, we also found a more profound DAT loss in the AC than in the VS; this feature provided the best diagnostic value for differentiating the PSP group from other groups. These results are consistent with a previous study showing that extensive pathologic degeneration of the pars compacta impaired the projection to the head of the caudate in PSP (31). However, in contrast to previous studies with ${ }^{123}$ I- $\beta$-CIT (31) and ${ }^{123}$ I-IPT SPECT (13), our study showed an anteroposterior gradient of DAT loss in patients with PSP that was similar to that in patients with PD and MSA. Technically, this finding was

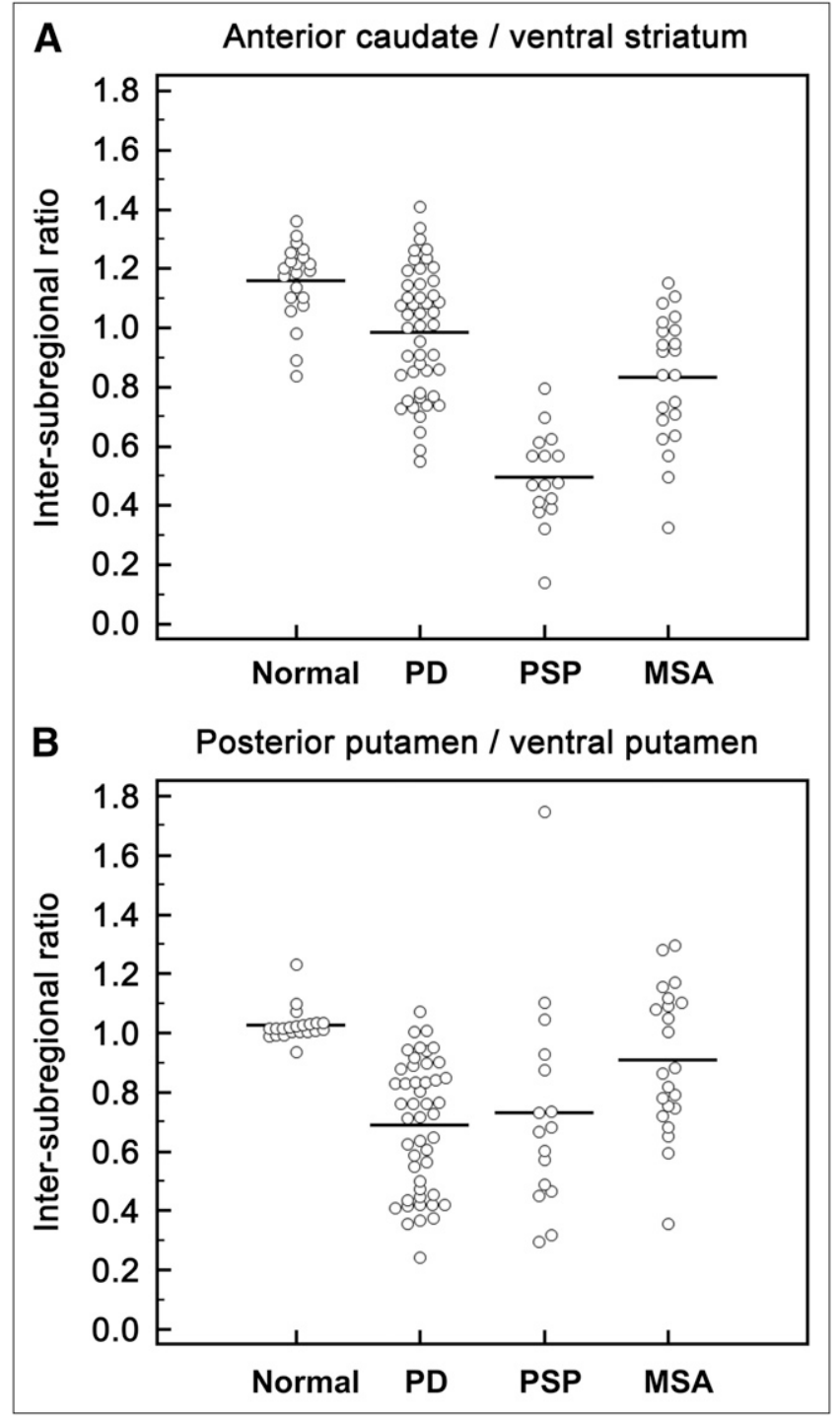

FIGURE 3. ISRS of $\mathrm{BP}_{\mathrm{ND}}$ values. (A) AC/VS ratios in PSP group were significantly lower than those in PD group, MSA group, and healthy controls. (B) PP/VP ratios in MSA group were significantly higher than those in PD group.

mainly due to the clearer separation of striatal subregions afforded by the increased spatial resolution of the PET camera compared with a SPECT camera (16). In other words, we reduced the spillover of higher putaminal ${ }^{123} \mathrm{I}-$ $\beta$-CIT uptake into the adjacent caudate head that led to an overestimation of the caudate signal in a previous study (32).

${ }^{18}$ F-FDG PET often missed small changes in striatal metabolism in early MSA because the nigrostriatal dopaminergic terminals comprise less than one-fifth of the striatal synapses (33). In contrast, in the present study, striatal DAT loss was evident in all patients with MSA despite the relatively short duration of symptoms.

Consistent with previous ${ }^{123}$ I- $\beta$-CIT studies $(34,35)$, the present study showed similar anteroposterior gradients in the MSA and PD groups. With respect to the ventrodorsal 
TABLE 5

Visual Interpretation of ${ }^{18} \mathrm{~F}-\mathrm{FP}-\mathrm{CIT}$ PET Images of Patients with PD, PSP, and MSA

\begin{tabular}{lrrr}
\hline & \multicolumn{3}{c}{$\begin{array}{c}\text { No. (\%) of patients with visual } \\
\text { diagnosis of: }\end{array}$} \\
\cline { 2 - 4 } $\begin{array}{c}\text { Clinical diagnosis } \\
\text { (no. of patients) }\end{array}$ & PD & PSP & MSA \\
\hline PD (49) & $46(94)$ & $2(4)$ & $1(2)$ \\
PSP (19) & $2(11)$ & $15(78)$ & $2(11)$ \\
MSA (24) & $5(21)$ & $3(12)$ & $16(67)$ \\
\hline
\end{tabular}

gradient, however, the MSA group showed a relatively even DAT loss from the VP to the PP; in contrast, the PD group showed a ventrodorsal gradient of putaminal DAT loss. In this context, we suggest that the ventrodorsal gradient is more useful for differentiating PD from MSA than the anteroposterior gradient, even in the early stage. The different patterns may reflect the different neuropathologic bases of these diseases. Goto et al. (36) reported that the loss of striatal dopaminergic neurons preceded nigral involvement in MSA, which is opposite that in PD (7), although the ventrolateral substantia nigra is known to be the most pathologically affected region in both PD and MSA. We believe that this early pathologic involvement of the striatum as well as the midbrain in MSA results in a relatively even loss of putaminal DAT without a ventrodorsal gradient.

The AC/VS and PP/VP ISRs showed good diagnostic accuracy for differentiating PSP and MSA from PD, respectively. In the present study, we chose the optimum cutoff probability values in a way that maximized sensitivity to complement the low sensitivity of clinical criteria because, in the clinical setting, ${ }^{18} \mathrm{~F}$-FP-CIT PET is used mainly as a confirmatory test for DAT loss in patients with suspected PD rather than for the differential diagnosis of APD.

Visual interpretation showed comparatively superior diagnostic accuracy for differentiating PSP or MSA from PD mainly because this approach could take into account not only ISRs but also other circumstances, such as asymmetry.

Finally, several methodologic and clinical limitations of the present study deserve mention. First, considering the current subcentimeter spatial resolution of a PET scanner and the sizes of the striatal subregions, partial-volume effects cannot be excluded. Given that nonlinear partial-volume effects lead to an underestimation of the concentration of radioactivity in small lesions (37), the quantification of signals in the posterior parts of the putamen likely would be particularly affected (32). In the present study, however, the ${ }^{18} \mathrm{~F}$-FP-CIT signals in such subregions in all groups of patients were significantly lower than those in healthy controls. Therefore, this potential issue is less likely to affect classification by ISRs.

Second, template-based VOI analysis might further reduce resolution during the normalization of individual PET images to the ${ }^{18} \mathrm{~F}$-FP-CIT PET template. Accurate segmentation of this structure is of great interest. Because of the improved resolution of PET instrumentation, subdividing the putamen into multiple parts was recently attempted in a VOI-based study (38). In addition, Jokinen et al. (17) reported the reliable detection of early PD by ratio analysis of $6-{ }^{18} \mathrm{~F}$-flouro-L-dopa uptake in striatal subregions. In the present study, however, spatially normalized ${ }^{18}$ F-FP-CIT PET images of some patients showed misregistration with predefined VOI templates and were ultimately excluded from the VOI analysis. This problem might be intrinsic to template-based analysis for patients with severe cerebral atrophy, ventricle enlargement, and advanced stages of parkinsonian disorders (39).

Third, we used ISRs to show different patterns of DAT loss in striatal subregions. Although this approach is simple, it might result in overestimation if there is a relatively severe loss at the bottom of the fraction. This scenario could explain the extraordinary asymmetry of the $\mathrm{PC}$ in the PSP group.

Finally, patients in the present study were diagnosed clinically. Therefore, misdiagnoses could have occurred because of the lack of postmortem verification (40). Although all diagnoses were based on strict diagnostic criteria, a longer period of follow-up is needed, especially given that the overdiagnosis of PD and the underdiagnosis of atypical parkinsonism are common (11).

Despite these limitations, the results of our study set the stage for the use of ${ }^{18} \mathrm{~F}-\mathrm{FP}$-CIT PET for the differential diagnosis of parkinsonian disorders. Analysis of the detailed subregions of the striatum provides insights into the pathophysiology of each disease. Moreover, the comparatively superior performance of visual interpretation adds the value of daily clinical practice for the detection and differentiation of parkinsonism.

\section{CONCLUSION}

PD, PSP, and MSA had significantly reduced striatal subregional $\mathrm{BP}_{\mathrm{ND}}$ values but different preferential subregional decreases in striatal DAT binding when examined by ${ }^{18} \mathrm{~F}-\mathrm{FP}-\mathrm{CIT}$ PET. Compared with PD, PSP and MSA had more prominent and earlier DAT loss in the AC and VP, respectively. These findings could be useful for suggesting PSP or MSA in parkinsonian cases without characteristic atypical features.

\section{DISCLOSURE STATEMENT}

The costs of publication of this article were defrayed in part by the payment of page charges. Therefore, and solely to indicate this fact, this article is hereby marked "advertisement" in accordance with 18 USC section 1734.

\section{ACKNOWLEDGMENT}

This work was supported by the real-time molecular imaging research program (No. 2010-002040) from the National Research Foundation (NRF). No other potential conflict of interest relevant to this article was reported. 


\section{REFERENCES}

1. Hughes AJ, Daniel SE, Lees AJ. The clinical features of Parkinson's disease in 100 histologically proven cases. Adv Neurol. 1993;60:595-599.

2. Jellinger KA. Pathology of Parkinson's disease: changes other than the nigrostriatal pathway. Mol Chem Neuropathol. 1991;14:153-197.

3. Bower JH, Dickson DW, Taylor L, et al. Clinical correlates of the pathology underlying parkinsonism: a population perspective. Mov Disord. 2002;17:910-916.

4. Litvan I, Agid Y, Jankovic J, et al. Accuracy of clinical criteria for the diagnosis of progressive supranuclear palsy (Steele-Richardson-Olszewski syndrome). Neurology. 1996;46:922-930.

5. Seppi K, Yekhlef F, Diem A, et al. Progression of parkinsonism in multiple system atrophy. J Neurol. 2005;252:91-96.

6. Barclay CL, Lang AE. Dystonia in progressive supranuclear palsy. J Neurol Neurosurg Psychiatry. 1997;62:352-356.

7. Wenning GK, Tison F, Ben Shlomo Y, et al. Multiple system atrophy: a review of 203 pathologically proven cases. Mov Disord. 1997;12:133-147.

8. Bensimon G, Ludolph A, Agid Y, et al. Riluzole treatment, survival and diagnostic criteria in Parkinson plus disorders: the NNIPPS study. Brain. 2009;132:156-171.

9. Braak H, Del Tredici K. Invited article: nervous system pathology in sporadic Parkinson disease. Neurology. 2008;70:1916-1925.

10. Litvan I, Bhatia KP, Burn DJ, et al. Movement Disorders Society Scientific Issues Committee report: SIC Task Force appraisal of clinical diagnostic criteria for parkinsonian disorders. Mov Disord. 2003;18:467-486.

11. Poewe W, Wenning G. The differential diagnosis of Parkinson's disease. Eur J Neurol. 2002;9(suppl 3):23-30.

12. Tang CC, Poston KL, Eckert T, et al. Differential diagnosis of parkinsonism: a metabolic imaging study using pattern analysis. Lancet Neurol. 2010;9:149-158.

13. Im JH, Chung SJ, Kim JS, Lee MC. Differential patterns of dopamine transporter loss in the basal ganglia of progressive supranuclear palsy and Parkinson's disease: analysis with $\left[{ }^{123} \mathrm{I}\right] \mathrm{IPT}$ single photon emission computed tomography. J Neurol Sci. 2006;244:103-109.

14. Pirker W, Asenbaum S, Bencsits G, et al. $\left[{ }^{123} \mathrm{I}\right]$ beta-CIT SPECT in multiple system atrophy, progressive supranuclear palsy, and corticobasal degeneration. Mov Disord. 2000;15:1158-1167.

15. Varrone A, Marek KL, Jennings D, et al $\left[{ }^{123} \mathrm{I}\right]$ beta-CIT SPECT imaging demonstrates reduced density of striatal dopamine transporters in Parkinson's disease and multiple system atrophy. Mov Disord. 2001;16:1023-1032.

16. Cherry SR. Fundamentals of positron emission tomography and applications in preclinical drug development. J Clin Pharmacol. 2001;41:482-491.

17. Jokinen $\mathrm{P}$, Helenius $\mathrm{H}$, Rauhala E, et al. Simple ratio analysis of ${ }^{18} \mathrm{~F}$-fluorodopa uptake in striatal subregions separates patients with early Parkinson disease from healthy controls. J Nucl Med. 2009;50:893-899.

18. Takáts A. Diagnostic criteria and differential diagnosis of Parkinson disease. Ideggyogy Sz. 2003;56:144-154.

19. Gilman S, Wenning GK, Low PA, et al. Second consensus statement on the diagnosis of multiple system atrophy. Neurology. 2008;71:670-676.

20. Lee SJ, Oh SJ, Chi DY, et al. One-step high-radiochemical-yield synthesis of $\left[{ }^{18}\right.$ F]FP-CIT using a protic solvent system. Nucl Med Biol. 2007;34:345-351.

21. Sun FT, Schriber RA, Greenia JM, et al. Automated template-based PET region of interest analyses in the aging brain. Neuroimage. 2007;34:608-617.

22. Mawlawi O, Martinez D, Slifstein M, et al. Imaging human mesolimbic dopamine transmission with positron emission tomography, I: accuracy and precision of $\mathrm{D}_{2}$ receptor parameter measurements in ventral striatum. J Cereb Blood Flow Metab. 2001;21:1034-1057.

23. Seibyl JP, Marek KL, Quinlan D, et al. Decreased single-photon emission computed tomographic $\left[{ }^{123} \mathrm{I}\right]$ beta-CIT striatal uptake correlates with symptom severity in Parkinson's disease. Ann Neurol. 1995;38:589-598.

24. Haber SN, Fudge JL, McFarland NR. Striatonigrostriatal pathways in primates form an ascending spiral from the shell to the dorsolateral striatum. J Neurosci. 2000;20:2369-2382.

25. Wang J, Zuo CT, Jiang YP, et al. ${ }^{18}$ F-FP-CIT PET imaging and SPM analysis of dopamine transporters in Parkinson's disease in various Hoehn \& Yahr stages. J Neurol. 2007;254:185-190.

26. Townsend DW. Dual-modality imaging: combining anatomy and function. J Nucl Med. 2008;49:938-955

27. Morrish PK, Sawle GV, Brooks DJ. Regional changes in $\left[{ }^{18} \mathrm{~F}\right]$ dopa metabolism in the striatum in Parkinson's disease. Brain. 1996;119:2097-2103.

28. Braak H, Tredici KD. Staging of brain pathology related to sporadic Parkinson's disease. Neurobiol Aging. 2003;24:197-211.

29. Kovacs GG, Milenkovic IJ, Preusser M, Budka H. Nigral burden of $\alpha$-synuclein correlates with striatal dopamine deficit. Mov Disord. 2008;23:1608-1612.

30. Murphy KE, Karaconji T, Hardman CD, Halliday GM. Excessive dopamine neuron loss in progressive supranuclear palsy. Mov Disord. 2008;23:607-610.

31. Messa C, Volonte MA, Fazio F, et al. Differential distribution of striatal [ $\left.{ }^{123} \mathrm{I}\right]$ beta-CIT in Parkinson's disease and progressive supranuclear palsy, evaluated with single-photon emission tomography. Eur J Nucl Med. 1998;25:12701276.

32. Scherfler C, Seppi K, Donnemiller E, et al. Voxel-wise analysis of [ $\left.{ }^{123} I\right]$ beta-CIT SPECT differentiates the Parkinson variant of multiple system atrophy from idiopathic Parkinson's disease. Brain. 2005;128:1605-1612.

33. Lyoo CH, Jeong Y, Ryu YH, et al. Effects of disease duration on the clinical features and brain glucose metabolism in patients with mixed type multiple system atrophy. Brain. 2008;131:438-446.

34. Kim YJ, Ichise M, Ballinger JR, et al. Combination of dopamine transporter and D2 receptor SPECT in the diagnostic evaluation of PD, MSA, and PSP. Mov Disord. 2002;17:303-312.

35. Brücke T, Asenbaum S, Pirker W, et al. Measurement of the dopaminergic degeneration in Parkinson's disease with $\left[{ }^{123} \mathrm{I}\right]$ beta-CIT and SPECT: correlation with clinical findings and comparison with multiple system atrophy and progressive supranuclear palsy. J Neural Transm Suppl. 1997;50:9-24.

36. Goto S, Matsumoto S, Ushio Y, Hirano A. Subregional loss of putaminal efferents to the basal ganglia output nuclei may cause parkinsonism in striatonigral degeneration. Neurology. 1996;47:1032-1036.

37. Cook GJ. Pitfalls in PET/CT interpretation. $Q \mathrm{~J} \mathrm{Nucl} \mathrm{Med} \mathrm{Mol} \mathrm{Imaging.}$ 2007;51:235-243.

38. Yagi S, Yoshikawa E, Futatsubashi M, et al. Progression from unilateral to bilateral parkinsonism in early Parkinson disease: implication of mesocortical dopamine dysfunction by PET. J Nucl Med. 2010;51:1250-1257.

39. Koch W, Radau PE, Hamann C, Tatsch K. Clinical testing of an optimized software solution for an automated, observer-independent evaluation of dopamine transporter SPECT studies. J Nucl Med. 2005;46:1109-1118.

40. Litvan I, Goetz CG, Jankovic J, et al. What is the accuracy of the clinical diagnosis of multiple system atrophy? A clinicopathologic study. Arch Neurol. 1997;54:937-944. 